RESEARCH

Real Analysis Exchange

Vol. 21(2), 1995-96, pp. 440-458

C. Freiling, Department of Mathematics, California State University, San

Bernardino, California, 92407-2397

B. S. Thomson’ Department of Mathematics, Simon Fraser University, B.C., Canada V5A 1S6

\title{
SCATTERED SETS, CHAINS AND THE BAIRE CATEGORY THEOREM
}

\begin{abstract}
A scattered set of real numbers can be exhibited in terms of endpoints of the components of some nondecreasing sequence of open sets (chain). We study this connection between scattered sets and chains providing characterizations of scattered sets, semiscattered sets, certain splattered sets and sets with countable closure. It is shown that any application of the Baire Category Theorem on the real line leads naturally to a chain of open sets and hence to an exceptional scattered set. Some applications of this fact are given.
\end{abstract}

\section{Introduction}

There are a number of instances in real analysis where an exceptional set turns out to be particularly small — not merely countable, but scattered. Proofs often fail to give any insight into this situation and the underlying geometric structure of scattered sets themselves may not be apparent. Frequently such sets arise in category arguments. If the Baire category theorem is expressed in a manner slightly different than usual the exceptional scattered sets can be clearly exhibited. We shall show that every application of the Baire category theorem leads to a chain of open sets and that the endpoints of the components of such chains always form a splattered set.

Recall that a set in a topological space is scattered if every nonempty subset has an isolated point. One sided versions for sets of real numbers have

\footnotetext{
Key Words: scattered set, Baire Category Theorem

Mathematical Reviews subject classification: Primary: 26A21. Secondary: 54E52

Received by the editors September 8, 1994

* Research supported in part by a grant from NSF

${ }^{\dagger}$ Research supported in part by a grant from NSERC
} 
been considered in the past: a set of reals is right (left) scattered if every nonempty subset has a point isolated on the right (left); any such set is called semi-scattered. (Note that these are precisely the scattered sets of the right (left) Sorgenfrey line.)

A set of reals is splattered if every nonempty subset has a point isolated on one side at least. A splattered set may be expressed as the union of a right scattered set and a left scattered set. Scattered sets may, similarly, be viewed as the intersection of a right scattered set and a left scattered set. It is well-known that scattered sets are countable; for a proof that semi-scattered sets are countable see [1].

The purpose of this article is to suggest a natural process of how these exceptional sets come about. We claim that the most natural way such sets arise is through a well ordered chain of open sets $G_{0} \subseteq G_{1} \subseteq G_{2} \subseteq \ldots$ In Section 2 and Section 3 we will explore the connection between such chains and the various types of scattered sets. In Section 5 we show that a chain of open sets naturally occurs with each application of the Baire category theorem on the real line. Thus any covering

$$
\mathbb{R}=\bigcup_{n=1}^{\infty} E_{n}
$$

yields an associated left-scattered, right-scattered, scattered and splattered set. In Section 6 we suggest that coverings of the form (1) naturally occur in analysis every time we consider a gauge function $\delta: \mathbb{R} \rightarrow \mathbb{R}^{+}$. Several examples are given where considerations of a naturally defined gauge leads (by the process outlined above) to a splattered exceptional set.

The companion article [2] that appears also in this issue of the Exchange shows how the gauge approach alone leads easily and naturally to proofs of the scattered nature of exceptional sets. For most readers the methods there will suffice. We feel, however, that the full picture emerges more clearly by the considerations of chains and Baire category presented here.

\section{Chains of Open Sets}

By a chain of open sets we will mean a well-ordered, possibly transfinite, sequence

$$
G_{0} \subseteq G_{1} \subseteq G_{2} \subseteq \ldots
$$

of open subsets of $\mathbb{R}$. Given such a chain (2) and an ordinal $\alpha$ let $R_{\alpha}$ denote the set of finite right hand endpoints of components of the set $G_{\alpha}$ and let $L_{\alpha}$ denote the set of finite left hand endpoints of components of the same set. An elementary argument shows that $R=\bigcup_{\alpha} R_{\alpha}$ is left scattered and that 
$L=\bigcup_{\alpha} L_{\alpha}$ is right scattered. For example to see that $R$ is left scattered consider any nonempty subset $A \subseteq R$. There is a least ordinal $\alpha$ such that $A \cap R_{\alpha} \neq \emptyset$. Then any point $x$ in $A \cap R_{\alpha}$ is the right endpoint of a component of $G_{\alpha}$. Because $\alpha$ is the least such ordinal $G_{\alpha}$ does not intersect $A$. Hence $x$ is isolated on the left in $A$. It follows that $R$ is left scattered.

We shall refer to $R$ as the associated left scattered set of the chain (2), to $L$ as the associated right scattered set of the chain and to $R \cap L$ and $R \cup L$ as the associated scattered set and the associated splattered set of the chain.

We will call a chain (2) regular if there is no infinite strictly increasing sequence $I_{\alpha_{1}} \subset I_{\alpha_{2}} \subset I_{\alpha_{3}} \subset \ldots$ where $I_{\alpha_{n}}$ is a component of $G_{\alpha_{n}}$.

Proposition 1 Let $R^{\prime}$ be an arbitrary left scattered set and $L^{\prime}$ be an arbitrary right scattered set. Then there is a regular chain whose associated sets $R$ and $L$ satisfy

(i) $R^{\prime} \subseteq R$ and $L^{\prime} \subseteq L$.

(ii) $R^{\prime} \cap L^{\prime}=R \cap L$.

(iii) $R \backslash R^{\prime}$ and $L \backslash L^{\prime}$ are scattered.

(iv) If $R^{\prime}=\emptyset$ then $L^{\prime}=L$ and if $L^{\prime}=\emptyset$ then $R^{\prime}=R$.

Proof. We assume that $R^{\prime} \cup L^{\prime}$ is infinite, since the construction is immediate if not. Fix a bijection $f: R^{\prime} \cup L^{\prime} \rightarrow \mathbb{Z}^{+}$. We will construct a transfinite sequence of triples $\left(x_{\alpha}, y_{\alpha}, z_{\alpha}\right)$ of real numbers with $y_{\alpha}$ strictly between $x_{\alpha}$ and $z_{\alpha}$. We let

$$
L_{\alpha}^{\prime}=\left\{x_{\beta}: \beta \leq \alpha \text { and } x_{\beta}<z_{\beta}\right\}
$$

$R_{\alpha}^{\prime}=\left\{x_{\beta}: \beta \leq \alpha\right.$ and $\left.z_{\beta}<x_{\beta}\right\}$

$I_{\alpha}$ is the open interval between $x_{\alpha}$ and $y_{\alpha}$.

$N_{\alpha}$ is the open interval between $x_{\alpha}$ and $z_{\alpha}$.

Our construction will be carried out in such a way that at each stage $\alpha$ the following requirements are met:

$\left(C_{1}\right)$ If $L^{\prime} \subseteq \bigcup_{\beta<\alpha} L_{\beta}^{\prime}$ and $R^{\prime} \subseteq \bigcup_{\beta<\alpha} R_{\beta}^{\prime}$ then the sequence stops. Otherwise either $x_{\alpha} \in\left(L^{\prime} \backslash \bigcup_{\beta<\alpha} L_{\beta}^{\prime}\right) \cap L_{\alpha}^{\prime}$ and we choose $x_{\alpha}<y_{\alpha}<z_{\alpha}$ or $x_{\alpha} \in\left(R^{\prime} \backslash \bigcup_{\beta<\alpha} R_{\beta}^{\prime}\right) \cap R_{\alpha}^{\prime}$ and we choose $z_{\alpha}<y_{\alpha}<x_{\alpha}$.

$\left(C_{2}\right)$ None of $x_{\alpha}, y_{\alpha}, z_{\alpha}$ are in $\bigcup_{\beta<\alpha} N_{\beta}$.

$\left(C_{3}\right)$ Neither of $y_{\alpha}, z_{\alpha}$ is in $L^{\prime} \cup R^{\prime}$.

$\left(C_{4}\right)\left|z_{\alpha}-x_{\alpha}\right|<\left(f\left(x_{\alpha}\right)\right)^{-1}$. 
$\left(C_{5}\right) L^{\prime} \cap N_{\alpha} \subseteq L_{\alpha}^{\prime}$ and $R^{\prime} \cap N_{\alpha} \subseteq R_{\alpha}^{\prime}$.

Let us assume that a transfinite sequence $\left\{\left(x_{\alpha}, y_{\alpha}, z_{\alpha}\right)\right\}$ has been constructed meeting these five requirements. We show how this constructs a regular chain (2) whose associated sets $R$ and $L$ satisfy the four assertions of the proposition.

We define the chain by writing $G_{\alpha}=\bigcup_{\beta \leq \alpha} I_{\beta}$. Let $R$ and $L$ denote the associated sets of the chain. By the requirement $C_{1}$ we have $L^{\prime}=\bigcup_{\beta} L_{\beta}^{\prime}$ and $R^{\prime}=\bigcup_{\beta} R_{\beta}^{\prime}$. By the requirement $\left(C_{2}\right)$ each $I_{\alpha}$ is a component of $G_{\alpha}$ and so $L_{\alpha}^{\prime} \subseteq L$. Therefore $L^{\prime} \subseteq L$. Similarly $R^{\prime} \subseteq R$ and we have proved (i) of the proposition.

We now check that the chain is regular. If there is a strictly increasing sequence $N_{\alpha_{1}} \subset N_{\alpha_{2}} \subset N_{\alpha_{3}} \subset \ldots$ then, by requirement $\left(C_{4}\right)$, the sequence $\left\{f\left(x_{\alpha_{n}}\right)\right\}$ is bounded. Then some $\alpha_{n}$ is repeated infinitely often which is impossible by requirement $\left(C_{1}\right)$. Therefore no such strictly increasing chain exists. Similarly there is no strictly increasing chain $I_{\alpha_{1}} \subset I_{\alpha_{2}} \subset I_{\alpha_{3}} \subset$ .... Therefore the chain $\left\{G_{\alpha}\right\}$ is regular as required. By this regularity and condition $\left(C_{2}\right)$ each component of a set $G_{\alpha}$ is either $I_{\alpha}$ itself or is already a component of some $G_{\beta}$ for $\beta<\alpha$. Therefore the intervals $\left\{I_{\alpha}\right\}$ are the only components of the chain $\left\{G_{\alpha}\right\}$.

We now prove statement (ii) of the proposition. Suppose that $x$ is an arbitrary point in $L \cap R$. Then $x$ must be a right endpoint of some $I_{\alpha}$ and a left endpoint of some $I_{\beta}$. By requirement $\left(C_{2}\right)$ this point $x$ cannot be both $y_{\alpha}$ and $y_{\beta}$. Otherwise $x=y_{\beta} \in\left(x_{\alpha}, z_{\alpha}\right)=N_{\alpha}$ so that, by $\left(C_{2}\right), \beta<\alpha$ and $x=y_{\alpha} \in\left(z_{\beta}, x_{\beta}\right)=N_{\beta}$ so that, again by $\left(C_{2}\right), \beta>\alpha$. Therefore $x$ must be either $x_{\alpha}$ or $x_{\beta}$ and, hence, $x \in L^{\prime} \cup R^{\prime}$. But then $x$ cannot be either $y_{\alpha}$ or $y_{\beta}$ by requirement $\left(C_{3}\right)$. Hence $x=x_{\alpha}=x_{\beta}$ and so $x \in L^{\prime} \cap R^{\prime}$. Thus we have proved that $L \cap R \subseteq L^{\prime} \cap R^{\prime}$. We have already established that $L^{\prime} \subseteq L$. and $R^{\prime} \subseteq R$ and so $L \cap R=L^{\prime} \cap R^{\prime}$ which is statement (ii) of the proposition.

We now prove statement (iii) of the proposition. Let $A$ be an arbitrary nonempty subset of $L \backslash L^{\prime}$; we shall find an isolated point. If $x_{\alpha} \notin L^{\prime}$ then, by requirement $\left(C_{1}\right), x_{\alpha} \in R^{\prime}$ and so, using again the fact that $R^{\prime} \subseteq R$, we have $x_{\alpha} \in R$. But we know by now that $L \cap R=L^{\prime} \cap R^{\prime}$ and so $x_{\alpha} \notin L$. Therefore $A$ contains none of the points $x_{\alpha}$. The only points in $A$ are points $y_{\alpha}$ where $z_{\alpha}<y_{\alpha}<x_{\alpha}$. Let $\alpha$ be the least ordinal for which $y_{\alpha} \in A$. The set $N_{\alpha}=\left(z_{\alpha}, x_{\alpha}\right)$ can certainly contain points $y_{\beta}$ of $L$ but none with $\beta>\alpha$ because of requirement $\left(C_{2}\right)$. Since $\alpha$ is minimal for points in $A$ we have $A \cap\left(z_{\alpha}, x_{\alpha}\right)=\left\{y_{\alpha}\right\}$ and accordingly $y_{\alpha}$ is isolated in $A$. This proves that $L \backslash L^{\prime}$ is scattered. Similarly it may be shown that $R \backslash R^{\prime}$ is scattered. This completes the proof of statement (iii) of the proposition.

Finally we prove statement (iv) of the proposition. If $R^{\prime}=\emptyset$ then, by requirement $C_{1}$, for each ordinal $\alpha$, we have $x_{\alpha}<y_{\alpha}<z_{\alpha}$ so that $I_{\alpha}=\left(x_{\alpha}, y_{\alpha}\right)$ 
with $x_{\alpha} \in L^{\prime}$. Thus $L \subseteq L^{\prime}$ and, since have already established that $L^{\prime} \subseteq L$, this means $L=L^{\prime}$. In a similar manner it can be shown that if $L^{\prime}=\emptyset$ then $R=R^{\prime}$. This proves statement (iv) as required.

We now turn to the construction of the transfinite sequence $\left\{\left(x_{\alpha}, y_{\alpha}, z_{\alpha}\right)\right\}$. At the first step we can easily choose $\left(x_{0}, y_{0}, z_{0}\right)$. For example take, if possible, $x_{0}<y_{0}<z_{0}$ with $x_{0} \in L^{\prime}$ and $\left(x_{0}, z_{0}\right]$ disjoint from $L^{\prime} \cup R^{\prime}$. If we make sure that $\left|z_{0}-x_{0}\right|$ is smaller than $f\left(x_{0}\right)^{-1}$ then we have satisfied $\left(C_{1}\right)-\left(C_{5}\right)$. If this cannot be done then choose $z_{0}<y_{0}<x_{0} \in R^{\prime}$ in the same way. Since $R^{\prime}$ is left scattered and $L^{\prime}$ is right scattered one of these is possible unless $R^{\prime} \cup L^{\prime}=\emptyset$ in which case a trivial chain will suffice.

We suppose then that $\left(x_{\alpha}, y_{\alpha}, z_{\alpha}\right)$ have been chosen for all $\alpha<\gamma$ in such a way that the five requirements $\left(C_{1}\right)-\left(C_{5}\right)$ hold. Let $L^{\prime \prime}=L^{\prime} \backslash \bigcup_{\alpha<\gamma} L_{\alpha}^{\prime}$ and $R^{\prime \prime}=R^{\prime} \backslash \bigcup_{\alpha<\gamma} R_{\alpha}^{\prime}$.

Claim If $x \in L^{\prime \prime}$ (resp. if $x \in R^{\prime \prime}$ ) then $x$ is not isolated on the right (left) from the set

$$
c\left(\bigcup_{\alpha<\gamma} N_{\alpha}\right) \cap c\left(L^{\prime} \cup R^{\prime}\right) .
$$

(Here $c(E)$ denotes the set complementary to E.)

Proof of Claim Suppose that $x \in L^{\prime \prime}$. (The proof if $x \in R^{\prime \prime}$ is similar.) Let $\alpha<\gamma$. By $\left(C_{5}\right) x$ cannot be in $N_{\alpha}$. By $\left(C_{3}\right) x$ is neither $y_{\alpha}$ nor $z_{\alpha}$. Then, since $x \notin L_{\alpha}^{\prime}, x$ cannot be a left hand endpoint of $N_{\alpha}$. By $\left(C_{2}\right)$ if $\alpha<\beta<\gamma$ and $N_{\alpha}, N_{\beta}$ overlap then $N_{\alpha} \subseteq N_{\beta}$; also, by the argument given above, there are no strictly increasing sequences

$$
N_{\alpha_{1}} \subset N_{\alpha_{2}} \subset N_{\alpha_{3}} \subset \ldots
$$

with each $\alpha_{n}<\gamma$. Therefore there exist points in $c\left(\bigcup_{\alpha<\gamma} N_{\alpha}\right)$ that are arbitrarily close to $x$ on the right. If $x$ is isolated on the right from $\bigcup_{\alpha<\gamma} N_{\alpha}$ then we are done since $L^{\prime} \cup R^{\prime}$ is countable. Otherwise $x$ is a limit point of the set $c\left(\bigcup_{\alpha<\gamma} N_{\alpha}\right) \cap\left\{z_{\alpha}: z_{\alpha}>x, \alpha<\gamma\right\}$. But $\left(C_{3}\right)$ implies that this is a subset of the set (3) and the claim is proved.

We now show how to choose the points $\left(x_{\gamma}, y_{\gamma}, z_{\gamma}\right)$. There are two cases to consider.

Case 1: There is an open interval $J$ which intersects $L^{\prime \prime}$ but does not intersect $R^{\prime \prime}$.

Case 2: Every open interval $J$ which intersects $L^{\prime \prime}$ also intersects $R^{\prime \prime}$. 
In Case 1 choose an open interval $J$ which intersects $L^{\prime \prime}$ but does not intersect $R^{\prime \prime}$. Since $L^{\prime \prime}$ is right scattered we choose a subinterval $(x, z) \subseteq J$ such that $(x, z] \cap R^{\prime \prime}=\emptyset$ and such that $[x, z] \cap L^{\prime \prime}=\{x\}$. We set $x_{\gamma}=x$ and, using the claim proved above, we choose $y_{\gamma}$ and $z_{\gamma}$ from the set (3) in such a way that $x_{\gamma}<y_{\gamma}<z_{\gamma}$ and $\left|z_{\gamma}-x_{\gamma}\right|<f\left(x_{\gamma}\right)^{-1}$.

In Case 2 every open interval which intersects $L^{\prime \prime}$ also intersects $R^{\prime \prime}$. If $L^{\prime \prime} \cup R^{\prime \prime}=\emptyset$ then the process terminates. Otherwise there is an open interval $J$ intersecting $R^{\prime \prime}$. Since $R^{\prime \prime}$ is left scattered we choose a subinterval $(z, x) \subseteq J$ such that $[z, x) \cap L^{\prime \prime}=\emptyset$ and such that $[z, x] \cap R^{\prime \prime}=\{x\}$. We set $x_{\gamma}=x$ and, once more using the claim proved above, we choose $y_{\gamma}$ and $z_{\gamma}$ from the set (3) in such a way that $z_{\gamma}<y_{\gamma}<x_{\gamma}$ and $\left|z_{\gamma}-x_{\gamma}\right|<f\left(x_{\gamma}\right)^{-1}$.

In either case the requirements $\left(C_{1}\right)-\left(C_{5}\right)$ now follow for $\alpha=\gamma$ and this completes the proof.

Corollary 2 Every set that is scattered (resp. left scattered, right scattered) is the associated scattered (resp. left scattered, right scattered) set of some chain.

\section{Complete Splattered Sets}

In the preceding section we have seen that every set that is scattered or scattered on just one side can be obtained from a chain of open sets. It is not true, however, that every splattered set is the associated splattered set of some chain. It is this fact that prompts the following definition.

Definition $3 A$ set $S$ is said to be a complete splattered set if for every nonempty subset $A \subseteq S$ there is an interval $(a, b)$ with $(a, b) \cap A=\emptyset$, so that at least one of $a$ or $b$ is in $A$ and the other is either $\pm \infty$ or else is in $S$.

It follows immediately from the definition of a splattered set that every complete splattered set is splattered. If the set $S$ of Definition 3 is also scattered (resp. left scattered, right scattered) then we shall say that $S$ is a complete scattered (resp. left scattered, right scattered) set. Not every splattered set is a complete splattered set; indeed not every scattered set is complete. For example let $S$ denote the centers of the intervals complementary to the Cantor ternary set. Any interval with endpoints in $S$ must contain further points of $S$. Thus while $S$ is evidently scattered it cannot satisfy the requirements of Definition 3. Briefly we say a set is complete if it satisfies Definition 3.

If we consider subsets with more than one element, then the isolating intervals can always be finite as the following proposition shows.

Proposition $4 A$ set $S$ is complete if and only if for every subset $A \subseteq S$ with more than one element, there is an interval $(a, b)$ with $(a, b) \cap A=\emptyset$ and such that at least one of $a, b$ is in $A$ and the other is in $S$. 
Proof. Let $S$ be complete and let $A \subseteq S$ have more than one element. Delete from $A$ its maximum and minimum elements, if such exist. Call the resulting set $A^{\prime}$. If $A^{\prime}$ is empty then we can take $a=\min A$ and $b=\max A$. If $A^{\prime}$ is nonempty and has again a maximum or minimum element then a choice can be easily made: for example if $a=\max A^{\prime}$ and $b=\max A$ exist then this interval $(a, b)$ has the required property. Thus we can reduce to the case where $A^{\prime}$ has no maximum or minimum. Since $S$ is complete, there is an interval $(a, b)$ such that $(a, b) \cap A^{\prime}=\emptyset$ and such that one of the points $a, b$ is in $A^{\prime}$ and the other in $S \cup \pm \infty$. If $b=+\infty$ then $a$ is a maximum and if $a=-\infty$ then $b$ is a minimum; hence neither of these points can be infinite since $A^{\prime}$ has no maximum or minimum. It follows that $(a, b) \cap A=\emptyset$ and at least one of $a$, $b$ is in $A$ and the other is in $S$. Conversely if the assertion of the proposition holds then $S$ is complete by definition.

This gives immediately the following corollary which will be useful later.

Corollary 5 Suppose $I_{1} \subseteq I_{2} \subseteq I_{3} \subseteq \ldots$ are open intervals whose union covers a set $S$ and such that, for each $n$, the set $S \cap I_{n}$ is complete. Then $S$ is complete.

We also have the following proposition showing that portions of complete sets are complete.

Proposition 6 If $S$ is complete and $I$ is any interval then $S \cap I$ is also complete.

Proof. Let $A$ be a nonempty subset of $S \cap I$. Since $A$ is also a nonempty subset of $S$ there is an interval $(a, b)$ with $(a, b) \cap A=\emptyset$, so that at least one of $a$ or $b$ is in $A$ and the other is either $\pm \infty$ or else is in $S$. This interval $(a, b)$ suffices, too, to establish that $S \cap I$ is also complete unless one of its endpoints fails to be in the interval $I$. But in that case replace the offending endpoint with $+\infty$ or $-\infty$ and we are done.

The removal of a complete subset from a complete scattered set may not produce a complete set. For example, inside each interval $I_{n}$ complementary to the Cantor ternary set choose three points $a_{n}<b_{n}<c_{n}$ with $b_{n}$ (as before) at the midpoint. Let $S_{1}$ denote the collection of all the points $a_{n}, b_{n}$ and $c_{n}$ and let $S_{2}$ denote the collection of all the points $a_{n}$ and $c_{n}$. Then $S_{1}$ and $S_{2}$ are complete scattered sets while $S=S_{1} \backslash S_{2}$ is not complete as we have seen. These observations can be checked directly or made to follow from Proposition 8 below characterizing complete sets as those associated with chains.

The formation of unions, on the other hand, does preserve completeness. 
Proposition 7 If $S_{1}$ and $S_{2}$ are complete so too is the union $S_{1} \cup S_{2}$.

Proof. Let $A$ be a nonempty subset of $S_{1} \cup S_{2}$. We wish to find an interval $(a, b)$ with $(a, b) \cap A=\emptyset$ so that at least one of $a$ or $b$ is in $A$ and the other is either $\pm \infty$ or else is in $S_{1} \cup S_{2}$. One of the sets $A \cap S_{1}$ or $A \cap S_{2}$ is nonempty; let us assume that $A \cap S_{1} \neq \emptyset$. Then, since $S_{1}$ is complete, there is an interval $\left(a^{\prime}, b^{\prime}\right)$ with $\left(a^{\prime}, b^{\prime}\right) \cap\left(A \cap S_{1}\right)=\emptyset$, at least one of $a^{\prime}$ or $b^{\prime}$ is in $A \cap S_{1}$ and the other is either $\pm \infty$ or else is in $S_{1}$.

If $A \cap S_{2} \cap\left(a^{\prime}, b^{\prime}\right)=\emptyset$ then $A \cap\left(a^{\prime}, b^{\prime}\right)=\emptyset$. We take $(a, b)=\left(a^{\prime}, b^{\prime}\right)$ and we are done. Suppose then that $A_{2}=A \cap S_{2} \cap\left(a^{\prime}, b^{\prime}\right) \neq \emptyset$. Since $S_{2}$ is complete, there is an interval $\left(a^{\prime \prime}, b^{\prime \prime}\right)$ with $\left(a^{\prime \prime}, b^{\prime \prime}\right) \cap A_{2}=\emptyset$, at least one of $a^{\prime \prime}$ or $b^{\prime \prime}$ is in $A_{2}$ and the other is either $\pm \infty$ or else is in $S_{2}$. If both $a^{\prime \prime}$ and $b^{\prime \prime}$ are in the interval $\left(a^{\prime}, b^{\prime}\right)$ then we may take $(a, b)=\left(a^{\prime \prime}, b^{\prime \prime}\right)$ and we are done. If $a^{\prime \prime} \in\left(a^{\prime}, b^{\prime}\right)$ and $b^{\prime \prime}>b^{\prime}$ then we take $(a, b)=\left(a^{\prime \prime}, b^{\prime}\right)$. If $b^{\prime \prime} \in\left(a^{\prime}, b^{\prime}\right)$ and $a^{\prime \prime}<a^{\prime}$ then we take $(a, b)=\left(a^{\prime}, b^{\prime \prime}\right)$.

Our main proposition in this section now follows. This shows that Definition 3 characterizes those splattered sets that are directly associated with some chain.

Proposition 8 A set is complete if and only if it is the associated splattered set of some (regular) chain.

Proof. Suppose that $S$ is complete. Then $S$ is countable. Fix a bijection

$$
f: S \rightarrow\left\{\frac{1}{2}, \frac{1}{4}, \frac{1}{8} \ldots\right\} .
$$

We define inductively a regular chain $\left\{G_{\alpha}\right\}$ of open sets so that $S$ is precisely its associated splattered set.

Set $G_{0}=\emptyset$ and suppose that $G_{\alpha}$ has already been defined. Denote by $R_{\alpha}$ the set of right endpoints of components of $G_{\alpha}$ and by $L_{\alpha}$ the set of left endpoints of components of $G_{\alpha}$. Suppose that $L_{\alpha} \cup R_{\alpha} \subseteq S$ and let $C_{\alpha}=R_{\alpha} \cup G_{\alpha} \cup L_{\alpha}$ and let $S_{\alpha}=S \backslash C_{\alpha}$. If $S_{\alpha}=\emptyset$ then the process terminates. Otherwise, since $S$ is complete and $S_{\alpha}$ a nonempty subset of $S$, there is an open interval $J_{\alpha}$ with at least one endpoint $a_{\alpha}$ in $S_{\alpha}$ and the other endpoint in $S \cup\{ \pm \infty\}$ and such that $J_{\alpha} \cap S_{\alpha}=\emptyset$. Then $J_{\alpha} \cap S \subseteq C_{\alpha}$.

If $J_{\alpha} \cap S=\emptyset$ we define $I_{\alpha}=J_{\alpha}$. If $J_{\alpha} \cap S \neq \emptyset$ we choose a subinterval $I_{\alpha} \subseteq J_{\alpha}$ with one endpoint $a_{\alpha}$, the other endpoint in the set $L_{\alpha} \cup R_{\alpha}$ and sufficiently close to $a_{\alpha}$ that

$$
\sum_{y \in I_{\alpha} \cap S} f(y)<f\left(a_{\alpha}\right) .
$$

Since $a_{\alpha}$ is not in the set $C_{\alpha}$ this is possible. 
In either case we write $G_{\alpha+1}=G_{\alpha} \cup I_{\alpha}$. At limit stages $\lambda$ we let $G_{\lambda}=$ $\bigcup_{\beta<\lambda} G_{\beta}$. This defines the chain. We now proceed to establish that this chain is regular and has $S$ for its associated splattered set.

First, note that neither endpoint of the interval $I_{\alpha}$ is in the set $G_{\alpha}$. Therefore $I_{\alpha}$ must be itself a component of $G_{\alpha+1}$. In fact $I_{\alpha}$ is exactly that component of $G_{\alpha+1}$ which is not a component of any $G_{\beta}$ for $\beta \leq \alpha$.

We can now show that the chain is regular. Suppose that

$$
I_{\alpha_{1}} \subset I_{\alpha_{2}} \subset I_{\alpha_{3}} \subset \ldots
$$

is strictly increasing. Then either $a_{\alpha_{1}} \in I_{\alpha_{3}}$ or $a_{\alpha_{2}} \in I_{\alpha_{3}}$ since our construction required that at each stage $a_{\alpha} \notin C_{\alpha}$. Therefore, by (4),

$$
\sum_{y \in I_{\alpha_{3}} \cap S} f(y)>2 \sum_{y \in I_{\alpha_{1}} \cap S} f(y)
$$

Since $\sum_{y \in S} f(y)=1$ it follows that any sequence (5) as above must be finite. Hence the chain $\left\{G_{\alpha}\right\}$ is regular and the only components in the chain are the intervals $\left\{I_{\alpha}\right\}$ added at each non-limit stage.

Consider now the associated splattered set of the chain, namely the finite elements of the set $\bigcup_{\alpha} L_{\alpha} \cup R_{\alpha}$. At each successor stage the process either terminates (if $S_{\alpha}=\emptyset$ ) or else a point $a_{\alpha} \in S_{\alpha}$ is placed in the set $L_{\alpha+1} \cup R_{\alpha+1}$ while $S_{\alpha} \cap G_{\alpha+1}=\emptyset$. It follows that $S \subseteq \bigcup_{\alpha} L_{\alpha} \cup R_{\alpha}$.

To establish the other direction in this set inclusion note that both endpoints of $I_{\alpha}$ were chosen to be in $S \cup\{ \pm \infty\}$. It follows that the finite elements of $\bigcup_{\alpha} L_{\alpha} \cup R_{\alpha}$ are in $S$ and consequently $S$ is the associated splattered set of our chain.

Conversely suppose that $S$ is the associated splattered set of a chain $\left\{G_{\alpha}\right\}$ so that $S=R \cup L$. Let $A$ be a nonempty subset of $S$. We shall exhibit the interval $(a, b)$ of Definition 3 in order to verify that $S$ is complete. There is a minimal ordinal $\alpha$ with

$$
A \cap\left(R_{\alpha} \cup L_{\alpha}\right) \neq \emptyset .
$$

Let $(a, b)$ be a component of $G_{\alpha}$ with one endpoint in the set (6). Then $a$ and $b$ are both in $S \cup\{ \pm \infty\}$ and one of them is in the set (6) and so also in $A$. Also $A \cap(a, b)=\emptyset$ for if it contains a point that point must be in $R_{\beta} \cup L_{\beta}$ for some $\beta<\alpha$ contradicting the choice of $\alpha$.

It follows from Proposition 1 that every set that is splattered (resp. scattered, right scattered, left scattered) is contained in a complete set of that type. One might have expected that there would be a minimal "completion" but this is not the case. One can remove any point from a complete set and the resulting set remains complete. 
Proposition 9 Let $S$ be a complete splattered set and let $x \in S$. Then $S \backslash\{x\}$ is also complete.

Proof. Let $A$ be a nonempty subset of $S \backslash\{x\}$. We wish to prove the existence of an interval $(a, b)$ with $(a, b) \cap A=\emptyset$, at least one of $a$ or $b$ is in $A$ and the other is either $\pm \infty$ or else is in $S \backslash\{x\}$.

Since $A$ is also a subset of $S$ and $S$ is complete there is, by definition, an interval $\left(a^{\prime}, b^{\prime}\right)$ with $\left(a^{\prime}, b^{\prime}\right) \cap A=\emptyset$, at least one of $a^{\prime}$ or $b^{\prime}$ is in $A$ and the other is either $\pm \infty$ or else is in $S$. If $x \neq a^{\prime}$ and $x \neq b^{\prime}$ then this interval $(a, b)=\left(a^{\prime}, b^{\prime}\right)$ suffices for verifying the completeness of $S \backslash\{x\}$. If $x=a^{\prime}$ then an adjustment is necessary. Suppose $x=a^{\prime}$ and that $A \cap\left(-\infty, a^{\prime}\right)=\emptyset$. Then we take the interval $(a, b)=\left(-\infty, b^{\prime}\right)$ as our verifying interval. Suppose that $x=a^{\prime}$ and $A \cap\left(-\infty, a^{\prime}\right) \neq \emptyset$. Then the set $A_{1}=A \cap\left(-\infty, a^{\prime}\right)$ is a nonempty subset of $S$ and so there must be an interval $\left(a_{1}, b_{1}\right)$ with $\left(a_{1}, b_{1}\right) \cap A_{1}=\emptyset$, at least one of $a_{1}$ or $b_{1}$ is in $A_{1}$ and the other is either $\pm \infty$ or else is in $S$. If $b_{1}<a^{\prime}$ then we take the interval $(a, b)=\left(a_{1}, b_{1}\right)$. If $b_{1} \geq a^{\prime}$ then we take the interval $(a, b)=\left(a_{1}, b^{\prime}\right)$. The case $x=b^{\prime}$ may be similarly handled.

\section{Sets of Countable Closure}

It is easy to check that a set with countable closure is scattered. A slight strengthening of the definition of a complete splattered set (Definition 3) serves to characterize the class of sets with countable closure. This reveals a close connection between these concepts and exhibits them as clearly belonging to the same general family of exceptional sets.

Proposition $10 A$ set $S$ has countable closure if and only if for every subset $A \subseteq S$ there is an interval $(a, b)$ with both $a$ and $b$ in $A \cup\{ \pm \infty\}$ so that $A \cap(a, b)=\emptyset$.

Proof. Let $S$ have countable closure and suppose that a subset $A \subseteq S$ fails to satisfy the condition of the proposition. Then $A$ must have more than two points and for every $x, y \in A$ with $x<y$ there is a $z \in A$ such that $x<z<y$. From this it follows that $\bar{A}$ is uncountable which is a contradiction.

Conversely suppose that $S$ has an uncountable closure and let $P$ be a perfect subset of that closure. Let $A$ be that subset of $S$ obtained by reducing $S$ in the following way. In each closed interval $I$ contiguous to $P$, if $I \cap S \neq \emptyset$ then we delete all but one element of $I \cap S$. If $I$ has an infinite endpoint then we delete all of $I \cap S$. Note that $P$ is a subset of $\bar{A}$. Then for any interval $(a, b)$ with $a, b \in A \cup\{ \pm \infty\}\}$ there must be points in $P \cap(a, b)$ and hence also points in $A \cap(a, b)$. Thus no interval $(a, b)$ as in the statement of the proposition can be found. 
We note that as in the definition of complete set the $\{ \pm \infty\}$ can be deleted if we restrict our attention to subsets with more than one point. The proof is identical to that of Proposition 4.

Corollary 11 Every set of countable closure is complete.

Proof. Compare Proposition 10 with Definition 3.

We recall that while the union of complete sets is complete (Proposition 7) there is no similar assertion for intersections and differences. If one of the sets has countable closure then more can be said.

Proposition 12 Let $S$ be a complete splattered set and let the set $C$ have countable closure. Then $S \cup C, S \cap C$ and $S \backslash C$ are complete.

Proof. The statement for the union follows from Proposition 7 and Corollary 11. The statement for the intersection follows from Corollary 11. We show that $S \backslash C$ is complete. For this it is enough to show that $S \backslash \bar{C}$ is complete since by Corollary 11 and Proposition 7 it follows then that

$$
S \backslash C=(S \backslash \bar{C}) \cup(S \cap(\bar{C} \backslash C))
$$

is complete too.

Since $S \cup C$ is nowhere dense we may partition the real line into infinitely many compact intervals $I$ with the endpoints of each interval in the complement of $\overline{S \cup C}$. By Corollary 5 and Proposition 7 it is enough to show that $(S \backslash \bar{C}) \cap I$ is complete for each such interval $I$. Fix one of the intervals $I$.

Let $G_{0}$ be the complement of $\bar{C}$. Inductively we define $G_{\alpha+1}$ as the union of $G_{\alpha}$ with the points isolated in $\bar{C} \backslash G_{\alpha}$ and, for limit ordinals $\lambda$, we let $G_{\lambda}=\bigcup_{\alpha<\lambda} G_{\alpha}$.

For each point $x$ in $\bar{C}$ there is a least ordinal $\alpha$ so that $x \in G_{\alpha}$. (This ordinal is known as the Cantor-Bendixson rank of $x$ and denoted $\operatorname{rank}(x)$.) If $\bar{C} \cap I=\emptyset$ then let us say that the rank of $\bar{C} \cap I$ is 0 , otherwise we call the rank of $\bar{C} \cap I$ the maximum of the ranks of the elements of $\bar{C} \cap I$. Such a maximum must exist since $\bar{C} \cap I$ is compact.

We complete the proof by arguing inductively on the rank of $\bar{C} \cap I$. Assume that $\bar{C} \cap I$ has rank $\alpha$ and that for every compact interval $I^{\prime}$ whose endpoints are in the complement of $\overline{S \cup C}$ and for which the rank of $\bar{C} \cap I^{\prime}$ is less than $\alpha$ the set $(S \backslash \bar{C}) \cap I^{\prime}$ is complete. There are only finitely many elements of $\bar{C} \cap I$ with rank exactly $\alpha$. We may use these to partition $I$ into finitely many intervals $I_{1}, I_{2}, \ldots I_{n}$. It is enough (by Proposition 7) to show that each set $(S \backslash \bar{C}) \cap I_{k}$ is complete. For this it is enough again to show that $(S \backslash \bar{C}) \cap \operatorname{int}\left(I_{k}\right)$ is complete. But each interval $\operatorname{int}\left(I_{k}\right)$ may be partitioned 
into infinitely many compact intervals $J$ with endpoints in the complement of $\overline{S \cup C}$. But, for each such interval $J,(S \backslash \bar{C}) \cap J$ is complete by the induction hypothesis. Therefore, by Corollary 5 and Proposition $7, S \backslash \bar{C} \cap \operatorname{int}\left(I_{k}\right)$ is complete and the proof is finished.

Sets of countable closure can also be characterized as the associated splattered sets of chains with special properties. Note that in the simplest case of a chain $G_{0} \subseteq G_{1} \subseteq G_{2} \ldots$ of expanding open intervals (i.e. each $G_{\alpha}$ is an interval) then the associated endpoints $R$ and $L$ are particularly simple: $R$ is well ordered by $<$ and $L$ is well ordered by $>$.

Proposition $13 A$ set $S$ has countable closure if and only if it is the associated splattered set of a chain $G_{0} \subseteq G_{1} \subseteq G_{2} \ldots$ where each set $\bigcup_{\beta<\alpha} G_{\beta}$ has only finitely many components.

Proof. We shall say a set $S$ is finitely splattered if it is the associated splattered set of a chain $\left\{G_{\alpha}\right\}$ such that for each $\alpha$ the open set $\bigcup_{\beta<\alpha} G_{\beta}$ has only finitely many components. If $I$ is an interval containing both $S$ and $\bigcup_{\alpha} G_{\alpha}$ then we shall say that $S$ is finitely splattered in $I$. We wish to show that any set of countable closure is finitely splattered.

Claim 1 If $S$ is finitely splattered and $I=(a, b)$ is any interval containing more than one point of $S$ then $S \cap I$ finitely splattered in $I$.

To prove this claim, let $S$ be the associated splattered set of a chain $\left\{G_{\alpha}\right\}$ which has the property that, for each $\alpha, \bigcup_{\beta<\alpha} G_{\beta}$ has only finitely many components. Consider the chain $\left\{G_{\alpha} \cap(a, b)\right\}$. If $a, b$ never appear as an endpoint of a component of an open set in this chain then we are done since this new chain exhibits $S \cap(a, b)$ as finitely splattered in $(a, b)$. Otherwise we have to amend the chain.

Suppose $S \cap(a, b)$ contains at least two points and that one at least of $a$, $b$ appears as an endpoint of a component of an open set in the chain $\left\{G_{\alpha}^{\prime}\right\}=$ $\left\{G_{\alpha} \cap(a, b)\right\}$. Let $(a, m)$ be the first component of $\left\{G_{\alpha}^{\prime}\right\}$ (i.e. with smallest ordinal) which uses $a$ as an endpoint and let $(n, b)$ be the first component using $b$.

Case 1: The number $m$ exists but not $n$. Since $S \cap(a, b)$ contains at least two points, we distinguish the following subcases:

Case 1a: There is a point $x$ in $S \cap(a, m)$. Then we let $n=m$ and reassign $m$ to be the value $x$ and go to case 3 .

Case 1b: There is a point $x$ in $S \cap(m, b)$. Then let $n=x$ and go to case 3 .

Case 2: The number $n$ exists but not $m$. This is handled in a similar way to case 1. 
Case 3: Both $m$ and $n$ exist and $m<n$. Let $\mathcal{C}_{1}$ be the chain $\left\{G_{\alpha} \cap(a, m)\right\}$ truncated before the interval $(a, m)$ occurs. Let $\mathcal{C}_{2}$ be the chain $\left\{G_{\alpha} \cap(m, n)\right\}$ with $(m, n)$ adjoined at the end. Let $\mathcal{C}_{3}$ be the chain $\left\{G_{\alpha} \cap(n, b)\right\}$ truncated before the interval $(n, b)$ occurs. By repeating sets if necessary, we may assume that $\mathcal{C}_{1}$ and $\mathcal{C}_{3}$ have the same length. Then form a chain $\mathcal{C}$ by adjoining to $\mathcal{C}_{2}$ the union of the chains $\mathcal{C}_{1}$ and $\mathcal{C}_{3}$.

Case 4: Both $m$ and $n$ exist and $m \geq n$. Then either $m=b$ or $n=a$. Then the interval $(a, b)$ occurs in this chain. By truncating the chain $\left\{G_{\alpha}^{\prime}\right\}$ before this occurs we destroy the fact that both $m$ and $n$ exist and hence this reduces to one of the previous cases.

We have now arrived at a chain that exhibits the fact that $S \cap(a, b)$ is finitely splattered in $(a, b)$ and so proving the claim.

Claim 2 If $S \cap(x, y)$ is finitely splattered and $x, y \in S$ then $S \cap[x, y]$ is finitely splattered in the interval $[x, y]$.

If $S \cap(x, y)$ is empty then the chain consisting of just the one open set $(x, y)$ works to prove the claim. If $S \cap(x, y)$ contains one point $z$ then the chain consisting of just the one open set $(x, z) \cup(z, y)$ works. If $S \cap(x, y)$ contains more than one point then there is a chain that exhibits the set $S \cap(x, y)$ as finitely splattered in $(x, y)$ using Claim 1. Adjoin to this chain the interval $(x, y)$.

Claim 3 Let $\ldots x_{-2}<x_{-1}<x_{0}<x_{1}<x_{2}<\ldots$ be a sequence of at least two points in $S$ which may be finite or infinite in each direction. Suppose that for each $k, S \cap\left[x_{k}, x_{k+1}\right]$ is finitely splattered in $\left[x_{k}, x_{k+1}\right]$ and suppose that the collection of intervals $\left\{\left[x_{k}, x_{k+1}\right]\right\}$ covers $S$. Then $S$ is finitely splattered.

To see this, for each $k$, let $\left\{G_{\alpha}^{k}\right\}$ be a chain that exhibits the set $S \cap$ $\left[x_{k}, x_{k+1}\right]$ as finitely splattered in $\left[x_{k}, x_{k+1}\right]$. Then the chain formed by $\left\{G_{\alpha}^{0}\right\}$ adjoined with $\left\{G_{\alpha}^{1} \cup\left(x_{0}, x_{1}\right)\right\}$, then adjoined with $\left\{G_{\alpha}^{-1} \cup\left(x_{0}, x_{2}\right)\right\}$, then adjoined with $\left\{G_{\alpha}^{2} \cup\left(x_{-1}, x_{2}\right)\right\}$ and so on in this manner will form a chain that demonstrates that $S$ is finitely splattered.

Claim 4 Let $a<b \leq c<d$ be such that $a \in S, d \in S$ and $S \cap(b, c)=\emptyset$. Suppose also that $S \cap(a, b)$ is finitely splattered in $(a, b)$ and $S \cap(c, d)$ is finitely splattered in $(c, d)$. Then $S \cap[a, d]$ is finitely splattered in $[a, d]$.

To see this let $\left\{G_{\alpha}\right\}$ be a chain that exhibits the set $S \cap(a, b)$ as finitely splattered in $(a, b)$ and $\left\{G_{\alpha}^{\prime}\right\}$ be a chain that exhibits the set $S \cap(c, d)$ as finitely splattered in $(c, d)$. By repeating open sets, if necessary, in either of the chains we can assume that $\left\{G_{\alpha}\right\}$ and $\left\{G_{\alpha}^{\prime}\right\}$ have the same length. Then we use the chain $\left\{G_{\alpha} \cup G_{\alpha}^{\prime}\right\}$ and adjoin

1. $(a, b) \cup(c, d)$ if both $b$ and $c$ are in $S$.

2. $(a, b) \cup(b, d)$ if $b \in S$ and $c \notin S$. 
3. $(a, c) \cup(c, d)$ if $b \notin S$ and $c \in S$.

4. $(a, d)$ if neither $b$ nor $c$ is in $S$.

Then the resulting chain exhibits that $S \cap[a, d]$ is finitely splattered in $[a, d]$.

Suppose now that $S$ has countable closure. We define the rank of the set $S$ as $\operatorname{rank}(S)=\bigcup_{x \in \bar{S}} \operatorname{rank}(x)$ where $\operatorname{rank}(x)$ is an ordinal denoting the CantorBendixson rank of $x$ as previously used in Proposition 12. We prove that $S$ is finitely splattered by induction on the rank. Since this is trivial for sets of rank 0 or 1 suppose that $S$ has rank $\beta>1$ and that any set of countable closure with rank smaller than $\beta$ is finitely splattered. Let $M=\{x \in \bar{S}: \operatorname{rank}(x)=\beta\}$. Then $M$ must be discrete.

Form a sequence of points ... $x_{-2}<x_{-1}<x_{0}<x_{1}<x_{2}<\ldots$ from the set $S \cup M$ which includes all of the set $M$ and such that the collection of intervals $\left\{\left[x_{i}, x_{i+1}\right]\right\}$ covers $S$ and such that if $x_{i}, x_{i+1} \in M$ then $S \cap\left(x_{i}, x_{i+1}\right)=\emptyset$ and such that in each interval $\left(x_{i}, x_{i+1}\right)$ the set $S$ is either empty or contains at least two points. If $x<y$ are any two points in $S \cap\left(x_{i}, x_{i+1}\right)$ then the induction hypothesis gives us that $S \cap(x, y)$ is finitely splattered. By Claim 2 then $S \cap[x, y]$ is finitely splattered in $[x, y]$. By Claim 3 we get that $S \cap$ $\left(x_{i}, x_{i+1}\right)$ is finitely splattered. By Claim $1, S \cap\left(x_{i}, x_{i+1}\right)$ is finitely splattered in $\left(x_{i}, x_{i+1}\right)$. On the other hand if $S \cap\left(x_{i}, x_{i+1}\right)$ is empty then, trivially, it is finitely splattered in $\left(x_{i}, x_{i+1}\right)$. If $x_{n} \in S$ and $m$ is the least integer greater than $n$ such that $x_{m} \in S \cup\{\sup S\}(m=n+1$ or $n+2)$ then, by Claim 4, $(S \cup\{\sup S\}) \cap\left[x_{n}, x_{m}\right]$ is finitely splattered in $\left[x_{n}, x_{m}\right]$. We may repeat this in the other direction so that, by Claim $3, S \cap[\inf S, \sup S]$ is finitely splattered. If inf $S \notin S$ then we may replace it with $-\infty$ in the chain and if $\sup S \notin S$ we may replace it with $\infty$. The resulting chain verifies that $S$ is finitely splattered. The proof now follows by induction.

Conversely let us suppose that $S$ is the associated splattered set of such a chain. Because each set $\bigcup_{\beta<\alpha} G_{\beta}$ has only finitely many components we can enlarge the chain (and hence the set $R \cup L$ ) by adding elements so that at limit ordinals $\lambda, G_{\lambda}=\bigcup_{\beta<\lambda} G_{\beta}$. We show that $R \cup L$ is closed in this case and it will follow, in general, that $R \cup L$ has countable closure.

We establish this by induction on the length of the chain. If the chain has length 1 then $R \cup L$ is finite and consequently closed as required. If the statement is true for any chain of length $\alpha$ then it is true for any chain of length $\alpha+1$ since $R_{\alpha+1} \cup L_{\alpha+1}$ is only finite.

It remains to consider limit ordinals $\lambda$. Suppose that the statement is true for any chain of length $\alpha<\lambda$ and that our chain has length $\lambda$. Let $\left\{x_{n}\right\}$ be a sequence of points in $R \cup L$ converging to a point $x$. We show that $x \in R \cup L$. There are ordinals $\alpha_{n}<\lambda$ so that $x_{n} \in R_{\alpha_{n}} \cup L_{\alpha_{n}}$. As $G_{\lambda}$ has only finitely many components either the sequence $\left\{x_{n}\right\}$ is eventually constant or else all but finitely many of the terms are in $G_{\lambda}$. We can assume then, without loss 
of generality, that all terms are in some component of $G_{\lambda}$. If $x$ is an endpoint of that component then $x \in R \cup L$ and we are done. If not then $x$ must be an interior point of that component and so belongs to some $G_{\alpha}$ for $\alpha<\lambda$. Eventually then all $x_{n}$ are also in $G_{\alpha}$ and so, by the induction hypothesis, $x \in R_{\alpha} \cup L_{\alpha} \subseteq R \cup L$ as we wished to prove.

\section{The Scattered Baire Category Theorem}

In this section we illustrate how chains of open sets (and hence complete splattered sets) arise from every application of the Baire Category Theorem on the real line. Suppose that we are given a cover of the reals

$$
\mathbb{R}=\bigcup_{i=1}^{\infty} E_{i}
$$

Then, by a single application of the Baire Category Theorem, there is a set $E_{i}$ dense in some open set $G_{0}$. Unless $G_{0}=\mathbb{R}$ this process would naturally repeat: there is a further member $E_{j}$ somewhere dense in $\mathbb{R} \backslash G_{0}$ so that there is a larger open set $G_{1}$ with $E_{j}$ dense in $G_{1} \backslash G_{0}$. This process can be continued transfinitely producing a chain of open sets covering the real line. We state this simple observation as a proposition. We suggest this name for the proposition to emphasize the role of the associated scattered and splattered sets that accompany any chain.

Proposition 14 (Scattered Baire Theorem) Let there be given a cover of the real line (7). Then there is a chain of open sets $\left\{G_{\alpha}\right\}$ whose union is all of $\mathbb{R}$ and such that for each $\alpha$ there is an index $i$ so that $E_{i}$ contains a dense subset of $G_{\alpha} \backslash \bigcup_{\beta<\alpha} G_{\beta}$.

Proof. Inductively define $G_{\alpha}=\left(\bigcup_{\beta<\alpha} G_{\beta}\right) \cup I$ where $I$ is an open interval containing points in

$$
\mathbb{R} \backslash\left(\bigcup_{\beta<\alpha} G_{\beta}\right)
$$

and such that some $E_{i}$ is dense in $I \backslash\left(\bigcup_{\beta<\alpha} G_{\beta}\right)$. The existence of such an interval is provided by the classical Baire Category Theorem as long as (8) is nonempty otherwise the process stops.

It is easy to see that any chain of open sets $\left\{G_{\alpha}\right\}$ whose union is $\mathbb{R}$ might arise from an application of the scattered Baire Theorem. For example one could let $E_{\alpha}=G_{\alpha} \backslash \bigcup_{\beta<\alpha} G_{\beta}$ and then renumber the transfinite sequence $\left\{E_{\alpha}\right\}$ as an ordinary sequence $\left\{E_{i}\right\}_{i=1}^{\infty}$. If Proposition 14 is then applied to the 
sequence $\left\{E_{i}\right\}$ then the original chain $\left\{G_{\alpha}\right\}$ could be recovered. Note however that the chain constructed by Proposition 14 is not necessarily unique. At any stage $\alpha$ we have the choice of adding one or several intervals. Also there may be more than one set $E_{i}$ that is somewhere dense in $\mathbb{R} \backslash \bigcup_{\beta<\alpha} G_{\beta}$ and to select $G_{\alpha}$ we would need to choose the right one. One might expect that this could be done in a more canonical fashion by adding the following condition (*) to the Scattered Baire Theorem:

for each index $\alpha$ there is an index $i$ such that

$$
G_{\alpha}=\left(\bigcup_{\beta<\alpha} G_{\beta}\right) \cup \bigcup\left\{(a, b): E_{i} \text { is dense in }(a, b) \backslash \bigcup_{\beta<\alpha} G_{\beta}\right\}
$$

and for each $j<i$ the set $E_{j}$ is nowhere dense in $\mathbb{R} \backslash \bigcup_{\beta<\alpha} G_{\beta}$.

The following example illustrates that doing so will limit the collection of chains that might be produced. Consider the chain of open sets defined by $G_{n}=\left(\frac{1}{n+1}, 2\right)$ for $n=0,1,2, \ldots$ and $G_{\omega}=(0,3)$. There cannot exist a sequence $E_{1}, E_{2}, E_{3}, \ldots$ such that for all $\alpha$ there is an index $i(\alpha)$ so that $(*)$ holds for $i=i(\alpha)$. If this were possible then each $i(\alpha)$ would be distinct. But then, for some integer $n$, we would have $i(\omega)<i(n)$ and yet $E_{i(\omega)}$ cannot be nowhere dense in $\mathbb{R} \backslash G_{n}$.

We can add some regularity conditions to the scattered Baire Theorem. Let us say that the chain of open sets $\left\{G_{\alpha}\right\}$ is dense if $G_{\alpha}$ is dense in

$$
\mathbb{R} \backslash \bigcup_{\beta<\alpha} G_{\beta}
$$

for each ordinal $\alpha$. The following variation of Proposition 14 gives a chain which is explicitly defined from the sequence $\left\{E_{i}\right\}$.

Proposition 15 Let there be given a cover of the real line (7). Then there is a dense chain of open sets $\left\{G_{\alpha}\right\}$ whose union is all of $\mathbb{R}$ and such that for each $\alpha$

$$
G_{\alpha}=\left(\bigcup_{\beta<\alpha} G_{\beta}\right) \cup \bigcup\left\{(a, b): \text { some } E_{i} \text { is dense in }(a, b) \backslash \bigcup_{\beta<\alpha} G_{\beta}\right\}
$$

ProOF. The proof is the same as for Theorem 14.

Given a dense chain of open sets $\left\{G_{\alpha}\right\}$ whose union is $\mathbb{R}$ we could again let $E_{\alpha}=G_{\alpha} \backslash \bigcup_{\beta<\alpha} G_{\beta}$ and then renumber $\left\{E_{\alpha}\right\}$ as an ordinary sequence 
$\left\{E_{i}\right\}_{i=1}^{\infty}$. Then $\left\{G_{\alpha}\right\}$ is the unique chain constructed by Proposition 15 from the sequence $\left\{E_{i}\right\}$. It is also possible to construct a sequence consisting of just two sets $\left\{E_{1}^{\prime}, E_{2}^{\prime}\right\}$ which yields, by Proposition 14 , the chain $\left\{G_{\alpha}\right\}$. One simply lets $E_{1}^{\prime}=\bigcup_{\alpha \text { odd }} E_{\alpha}$ and $E_{2}^{\prime}=\bigcup_{\alpha \text { even }} E_{\alpha}$.

\section{Applications}

We begin with a simple application, a variation of the Cantor-Bendixson theorem. Our proof is not much different than the original but it demonstrates the method of using chains to collect the scattered sets.

Theorem 16 (Cantor-Bendixson) Every set can be partitioned into four pieces, the first scattered, the second left scattered and with no isolated points, the third right scattered and with no isolated points, and the fourth set has only bilateral limit points.

Proof. Let $E$ be an arbitrary set. We define a chain $\left\{G_{\alpha}\right\}$ inductively. Start with $G_{0}=\emptyset$. At limit ordinals $\lambda$, we let $G_{\lambda}=\bigcup_{\alpha<\lambda} G_{\alpha}$. If $\alpha$ is not a limit ordinal then consider the set $E_{\alpha}=E \backslash\left(\bigcup_{\beta<\alpha} L_{\beta} \cap \bigcup_{\beta<\alpha} R_{\beta}\right)$ where, as usual, $L_{\alpha}$ denotes the set of left endpoints of components of $G_{\alpha}$ and $R_{\alpha}$ the set of right endpoints of components of $G_{\alpha}$.

If $E_{\alpha}$ contains a point $x$ isolated on the right then there is a $b_{\alpha}$ so that $E_{\alpha} \cap\left[x, b_{\alpha}\right]=\{x\}$. In that case we write $G_{\alpha}=\bigcup_{\beta<\alpha} G_{\beta} \cup\left(x, b_{\alpha}\right)$. If not and $E_{\alpha}$ contains a point $x$ isolated on the left then there is a $b_{\alpha}$ so that $E_{\alpha} \cap\left[b_{\alpha}, x\right]=\{x\}$. In that case we write $G_{\alpha}=\bigcup_{\beta<\alpha} G_{\beta} \cup\left(b_{\alpha}, x\right)$. If neither is so then $E_{\alpha}$ has no point isolated on either side and the process can stop.

Let $R, L$ be the associated right scattered and left scattered sets of the chain. Then $S_{1}=E \cap L \cap R$ is scattered, $S_{2}=E \cap L \cap c(R)$ is right scattered, $S_{3}=E \cap R \cap c(L)$ is left scattered and $S_{4}=E \cap c(L) \cap c(R)$ has no point that is isolated on either side. These four sets are clearly disjoint and $S_{2} \cup S_{3} \cup S_{4}$ has no isolated points. The sets $S_{2}$ and $S_{3}$, however, may have isolated points. Repeat this process on $S_{2}$ obtaining four sets $S_{21}, S_{22}, S_{23}$ and $S_{24}$ and repeat the process on $S_{3}$ obtaining $S_{31}, S_{32}, S_{33}$ and $S_{34}$. Then $S_{22} \cup S_{23} \cup S_{24}$ and $S_{32} \cup S_{33} \cup S_{34}$ have no isolated points. Let

$$
\begin{aligned}
& S_{1}^{\prime}=S_{1} \cup S_{21} \cup S_{31}, \\
& S_{2}^{\prime}=S_{22} \cup S_{23} \cup S_{24}, \\
& S_{3}^{\prime}=S_{32} \cup S_{33} \cup S_{34}, \\
& S_{4}^{\prime}=S_{4} .
\end{aligned}
$$


Note that these four sets partition $E$ since

$$
E=S_{1} \cup S_{2} \cup S_{3} \cup S_{4}=S_{1}^{\prime} \cup S_{2}^{\prime} \cup S_{3}^{\prime} \cup S_{4}^{\prime} .
$$

Clearly $S_{1}^{\prime}$ is scattered since it is a union of scattered sets. The set $S_{2}^{\prime}$ is right scattered, since it is a subset of $S_{2}$, and has no isolated points. The set $S_{3}^{\prime}$ is left scattered, since it is a subset of $S_{3}$, and has no isolated points. Finally $S_{4}^{\prime}=S_{4}$, as already observed, has no point isolated on either side. Hence the four sets $S_{1}^{\prime}, S_{2}^{\prime}, S_{3}^{\prime}, S_{4}^{\prime}$ form the required partition of $E$.

Of course the theorem can also be proved in a nonconstructive way, avoiding the introduction of chains. The referee has supplied the following sketch. Take $S_{4}$ as the largest bilaterally dense-in-itself subset of $E$, take $S_{3}$ as the largest subset of the (splattered) set $E \backslash S_{4}$ which is dense-in-itself from the left, and take $S_{2}$ as $E \backslash\left(S_{3} \cup S_{4}\right)$. Note that $S_{3}$ is right scattered and that $S_{2}$ is left scattered. Take $S_{2}^{\prime}$ (resp. $S_{3}^{\prime}$ ) to be the largest dense in itself subset of $S_{2}$ (resp. $S_{3}$ ). Collect the deleted points in a set $S_{1}$. Then the four sets $S_{1}$, $S_{2}^{\prime}, S_{3}^{\prime}, S_{4}$ form the required partition of $E$.

Our second application illustrates a useful connection between gauges, chains and scattered sets. Any gauge function (that is a map $\delta: \mathbb{R} \rightarrow \mathbb{R}^{+}$) leads naturally to a cover of the real line by using $E_{i}=\left\{x: \delta(x)>i^{-1}\right\}$. If a gauge is undefined on a countable set $N=\left\{z_{1}, z_{2}, z_{3}, \ldots\right\}$ then let

$$
E_{i}=\left\{z_{1}, z_{2}, \ldots, z_{i}\right\} \cup\left\{x: \delta(x)>i^{-1}\right\} .
$$

In any case we have $\mathbb{R}=\bigcup_{i=1}^{\infty} E_{i}$. This covering then naturally leads, by the scattered Baire Theorem, to an associated chain of open sets and, hence, to an associated scattered, right scattered, and left scattered set. This natural process is summed up by the following proposition.

Proposition 17 Let $\delta$ be a gauge defined on all of $\mathbb{R}$ except possibly for some countable set. Then, except for a right (left) scattered set, every point $x$ is the limit from the right (left) of some sequence $\left\{x_{i}\right\}$ for which $\delta\left(x_{i}\right)$ is bounded above zero.

Proof. We suppose that $\delta(x)$ is defined and positive at every point excepting for $x$ in a countable set $N=\left\{z_{1}, z_{2}, z_{3}, \ldots\right\}$. Write

$$
E_{i}=\left\{z_{1}, z_{2}, \ldots, z_{i}\right\} \cup\left\{x: \delta(x)>i^{-1}\right\} .
$$

Apply the scattered Baire Theorem to $\left\{E_{i}\right\}$ using unions at the limit ordinals to produce a chain $\left\{G_{\alpha}\right\}$ whose union is all of $\mathbb{R}$ and with the property that for each $\alpha$ there is an index $i$ such that $E_{i}$ contains a dense subset of $G_{\alpha} \backslash \bigcup_{\beta<\alpha} G_{\alpha}$. We show that every point $x$ not in the right scattered set $L$ associated with 
the chain is the limit from the right of some sequence $\left\{x_{i}\right\}$ for which $\delta\left(x_{i}\right)$ is bounded above zero.

Fix $x$, let $\eta$ be the least ordinal such that $x \in G_{\eta}$ and choose $i=i(\eta)$ such that $E_{i}$ contains a dense subset of $G_{\eta} \backslash \bigcup_{\beta<\eta} G_{\beta}$. If $x$ is a right hand limit point of $E_{i}$ then it is also a right hand limit point of the set

$$
\left\{x \in \mathbb{R} \backslash N: \delta(x)>i^{-1}\right\}
$$

and we are done. Otherwise $x$ is isolated on the right from $E_{i}$ and hence also from $G_{\eta} \backslash \bigcup_{\beta<\eta} G_{\beta}$. Choose $\epsilon>0$ sufficiently small so that $(x, x+\epsilon) \subseteq G_{\eta}$ and so that $(x, x+\epsilon)$ is disjoint from $G_{\eta} \backslash \bigcup_{\beta<\eta} G_{\beta}$. Then $(x, x+\epsilon) \subseteq \bigcup_{\beta<\eta} G_{\beta}$ and so $x$ is a left endpoint of a component of $\bigcup_{\beta<\eta} G_{\beta}$ and $x \in L$ as claimed.

For a direct proof of Proposition 17 without invoking the Scattered Baire Theorem see the companion article [2]. There too further applications are given and proved using the gauge approach alone.

\section{References}

[1] C. Freiling, Symmetric derivates, scattered and semi-scattered sets, Trans. American Math. Soc., 318 (1990), 705-720.

[2] C. Freiling and B. S. Thomson, Scattered Sets and Gauges, Real Analysis Exch., this issue.

[3] Z. Semadeni, Sur les ensembles clairsemés, Dissert. Math., 19 (1959), 39. 